Canadian Oncology

Nursing Journal

Revue canadienne

de soins infirmiers

en oncologie

Volume 29, Issue 3 • Summer 2019

elSSN: 2368-8076 


\title{
Les soins aux survivants du cancer: vers une grande qualité, en toute équité
}

\author{
par Tracy L. O. Truant, Colleen Varcoe, Carolyn C. Gotay et Sally Thorne
}

\section{RÉSUMÉ}

Les modèles de soins aux survivants du cancer évoluent rapidement, mais des disparités en matière de santé sont néanmoins de plus en plus évidentes entre les survivants de la maladie. Le Canada n'y échappe pas, et on observe que certains survivants privilégiés reçoivent des soins de plus grande qualité et conservent donc une meilleure santé que d'autres. La présente étude a pour but d'améliorer les soins aux survivants en aidant les cliniciens et les décideurs à mieux comprendre comment le vécu des survivants, les stratégies de gestion des soins et différents facteurs psychologiques et politiques peuvent influencer l'offre de soins aux survivants de grande qualité développés au Canada, ainsi que l'accès à ces soins. La présente étude descriptive et interprétative en trois étapes se fonde sur une approche infirmière épistémologique adoptant une perspective tant critique que multidimensionnelle. Nous avons procédé à une analyse textuelle critique de sources documentaires, de même qu'à une analyse secondaire de transcriptions d'entrevues provenant d'une base de données déjà existante; nous avons également mené des entrevues qualitatives auprès de 34 survivants et de 12 intervenants du système de santé. Nous avons ensuite extrait de ces données les facteurs individuels, collectifs et systémiques qui contribuent à creuser le fossé entre l'expérience de soins attendue et le vécu réel des survivants. En comprenant ce qui fait varier les soins aux survivants et les ressources qui leur sont allouées, nous espérons contribuer à éclaircir cette question complexe et encourager les cliniciens et les décideurs à adopter des approches à plusieurs niveaux pour assurer l'équité des soins de grande qualité.

\section{AU SUJET DES AUTEURES}

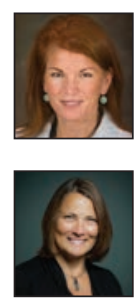

Tracy L.O. Truant, RN, PhD

Directrice ancienne, Recherche, Éducation et Innovation

BC Cancer, Vancouver BC

tracy.truant@bccancer.ca

Colleen Varcoe, RN, PhD, FCAHS,

Professeure, École des sciences infirmières

University of British Columbia

colleen.varcoe@ubc.ca



Carolyn C. Gotay, PhD, FCAHS

Professeure émérite, School of Population and Public Health

University of British Columbia

carolyn.gotay@ubc.ca

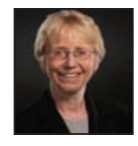

Sally Thorne, RN, PhD, FAAN, FCAHS

Professeure, École des sciences infirmières

University of British Columbia

sally.thorne@nursing.ubc.ca

Auteure-ressource : Sally Thorne

Sally.thorne@nursing.ubc.ca

1-604-822-7482

DOI: $10.5737 / 23688076293163169$

\section{CONTEXTE}

$\Lambda \mathrm{u}$ cours des vingt dernières années, plus de gens que $A_{\text {jamais ont vu leur espérance de vie augmenter après un }}$ diagnostic de cancer grâce aux progrès réalisés dans le dépistage et le traitement (Comité consultatif des statistiques canadiennes sur le cancer, 2018). Pour au moins les deux tiers des Nord-Américains ayant reçu un diagnostic de cancer, la question n'est plus de savoir s'ils vont survivre à la maladie, mais plutôt s'ils auront une bonne qualité de vie, ce qui les amène à se voir davantage comme des survivants que des victimes. (Bell, 2010). Or, nombreux sont les survivants du cancer qui, bien que techniquement guéris ou en rémission prolongée après leurs traitements, vivent des difficultés physiques, psychologiques, sexuelles, sociales et financières; la crainte persistante d'une récidive et les inquiétudes liées à la prévention secondaire, notamment, peuvent nuire à leur qualité de vie de manière permanente (Partenariat canadien contre le cancer [PCCC], 2012; Jones et Grunfeld, 2011; Mehnert, de Boer et Feuerstein, 2013; Ness et al., 2013). Les structures de soins officielles actuellement en place, ancrées dans un modèle biomédical axé sur le traitement et le contrôle de la maladie (soins du cancer) et sur le maintien du bien-être (soins de première ligne), présentent des lacunes importantes et ne permettent pas de répondre à la multitude de besoins des survivants en matière de soutien et de soins médicaux (PCCC, 2018; Wittal, 2018).

Il existe un large consensus selon lequel les modèles actuels de soins des survivants au cancer sont sous-optimaux partout au Canada, et ailleurs aussi (Alliance canadienne pour la recherche sur le cancer [ACRC], 2017; National Academies of Sciences, Engineering and Medicine, 2018). La coordination des soins spécialisés et des soins de première ligne manque de cohérence et les efforts se concentrent sur la surveillance médicale aux dépens du soutien psychologique et de la promotion de la santé (Fitch et al., 2018 sous presse; Shaw et JenningsShaw, 2017). Lorsqu'il est question de planification, on pense rarement à entendre l'opinion des survivants, et les groupes marginalisés, qui vivent des situations particulières en raison de leur marginalisation, sont communément absents du débat (Ashing-Giwa et al., 2013; Boehmer, Glickman, Milton et Winter, 2012; Brooks, 2010; ACRC, 2017; Gifford, Thomas, Barton, Grandpierre et Graham, 2018; Skinner, 2012).

La situation est rendue encore plus complexe par l'évolution du discours public sur la « survivance », discours souvent positif et peu nuancé, qui associe la rémission du cancer à une expérience « transformatrice » et valorise l'autosoin, les choix personnels et l'autonomie (Bell, 2012; Kromm, Smith et Singer, 2007). Ce genre de discours suscite des attentes irréalistes, en plus de faire peser la responsabilité de la guérison sur des personnes qui subissent encore parfois les effets 
difficiles et persistants de la maladie, notamment les patients défavorisés par les déterminants sociaux de la santé (Bell et Ristovski-Slijepcevic, 2013; Sinding, 2014).

Le Partenariat canadien contre le cancer (PCCC) a établi une stratégie pancanadienne dont le but est de fournir à tous les Canadiens un accès à des soins du cancer équitables, axés sur la personne et de grande qualité (2018). Pour ce faire, les ressources doivent être sûres, efficaces et équitables, disponibles en temps opportun et alignées sur les besoins des survivants afin d'optimiser les résultats souhaités, notamment une amélioration du taux de survie, des capacités fonctionnelles, de la qualité de vie et de l'expérience personnelle par rapport au cancer (Feuerstein et Ganz, 2011; Lotfi-Jam et al., 2009; Malin et al., 2011). Toutefois, l'absence de structures ou de contextes encourageant la prestation de soins axés sur la personne au sein des systèmes de santé et de soins du cancer peut mener à un accroissement involontaire des disparités en matière de santé, au détriment, particulièrement, des personnes marginalisées (Epstein, Fiscella, Lesser et Stange, 2010; Hankivsky, 2011; Niu, Roche, Pawlish et Henry, 2013; Weaver, Geiger, Lu et Case, 2013).

Pour optimiser la santé et le bien-être et atteindre l'équité, il faut s'assurer de mettre les besoins des personnes, et non leur statut social, au centre du processus. (Ahmed et Shahid, 2012). Les infirmières sont les mieux placées pour défendre les droits sociopolitiques des patients dans leur pratique, sans compter qu'elles en ont aussi l'obligation morale (Falk-Rafael, 2005). Les infirmières en oncologie sont convaincues de la nécessité, pour des raisons d'éthique et de justice sociale, de promouvoir et favoriser une santé optimale, à la fois pour les individus et les collectivités (Association canadienne des infirmières en oncologie [CANO/ACIO], 2006; Association des infirmières et infirmiers du Canada, 2017).

Si rien n'est fait, les modèles de soins actuels continueront de renforcer le mode de création, de mise en place et d'évaluation des ressources destinées aux survivants en vigueur sans jamais rectifier les inégalités (Jones et Grunfeld, 2011; Smith et Hall, 2015). Pour orienter notre réflexion sur les futurs modèles et ressources de soins aux survivants, il faudra mieux comprendre le contexte et tenir compte de la complexité des relations et croisements entre les différents facteurs qui influencent la capacité d'agir des survivants, que ce soit à l'échelle individuelle ou collective. Par conséquent, la présente étude vise plus qu'une simple description des enjeux et des défis : son objectif est de proposer des voies à explorer, des recommandations ou des principes d'action qui embrassent toute cette complexité, afin d'aménager des soins et des modèles de soins à la fois équitables et complets pour tous les survivants.

\section{QUESTION DE RECHERCHE}

La question directrice fondamentale de l'étude est la suivante : Comment les différents points de vue des survivants du cancer et des intervenants du système de santé peuventils éclairer une démarche visant à assurer aux survivants du cancer des soins de grande qualité en toute équité, partout au Canada? Pour répondre à cette question, nous avons cherché à savoir comment différents survivants du cancer décrivaient et expliquaient leurs expériences, notamment leur accès aux soins, de même que leur vision d'un système de soins adapté à leurs besoins; nous avons tenté de dégager des approches à partir des témoignages des survivants et des intervenants du système de soins du cancer pour appuyer la création de ressources et de modèles de soins de grande qualité qui répondent aux besoins individuels et collectifs.

\section{DEVIS DE L'ÉTUDE}

Le raisonnement théorique de la présente étude repose sur une approche pragmatique adoptant un point de vue critique multidimensionnel pour expliquer la complexité inhérente aux soins des survivants. Grâce à la description interprétative (une méthode quantitative appliquée), nous avons pu puiser dans l'épistémologie de la discipline infirmière pour élaborer une série de sous-études visant à générer, une fois mises en commun, de nouvelles pistes de compréhension qui permettront de transposer les phénomènes pertinents à la pratique clinique (Thorne, 2016; Thorne, Stephens et Truant, 2016).

Dans ce contexte, nous avons conçu une approche en trois étapes afin de laisser place à une diversité de points de vue, en procédant à une analyse de plus en plus complexe : nous avons d'abord réalisé une analyse textuelle critique, puis une analyse secondaire des transcriptions d'une base de données d'entrevues déjà constituée, avant de mener de nouvelles entrevues auprès de survivants du cancer et d'intervenants du système de santé. Les deux dernières étapes nécessitaient l'approbation du comité d'éthique de la recherche comportementale de notre université (UBC BREB no H09-0171 et no H14-0382). Il va sans dire que le détail de la méthodologie et des résultats de chaque étape ne peut être couvert en un seul article; chacune mériterait à elle seule un rapport complet. D'autres publications sont d'ailleurs prévues. L'objectif du présent article est donc de décrire le devis général utilisé et de présenter les résultats obtenus dans leurs grandes lignes. Les recommandations formulées pour améliorer les soins découlent de l'analyse combinée de ces trois processus à la fois distincts et interdépendants.

\section{Processus et résultats de l'étude \\ Revue critique de la documentation}

Nous avons, dans un premier temps, effectué une analyse textuelle critique de plus de 70 sources documentaires, notamment de lignes directrices sur la survivance, de programmes d'éducation, de politiques et de ressources, publiées au Canada pour la plupart. Certaines lignes directrices cliniques américaines ont été incluses à l'analyse, étant donné qu'elles influencent souvent les soins aux survivants d'ici en l'absence de lignes directrices canadiennes (pour des exemples, voir Resnick et al., 2015). Nous avons également choisi des documents portant plus exactement sur le soin des survivants en Colombie-Britannique, comme le cadre de soins axés sur le patient (Patient-Centred Care Framework, ministère de la Santé de la Colombie-Britannique, 2015) pour prendre en compte la région géographique des survivants interrogés pendant la troisième étape. Nous avons pu, grâce aux sources documentaires, brosser le portrait des difficultés, lacunes et 
incohérences actuelles, mais aussi relever des occasions de cerner et de renforcer des facteurs qui favoriseront l'équité et la qualité des soins aux survivants.

Ce corpus nous a permis de constater à quel point la définition du terme survivance varie selon les contextes. Par exemple, pour plusieurs groupes de défense des droits et organismes nationaux de lutte contre le cancer, la survivance s'étend du diagnostic à la fin de vie du patient (voir entre autres le PCCC, 2012; Ristovski-Slijepcevic, 2008; Réseau universitaire de santé, sans date). Des définitions intermédiaires la font commencer à la fin du traitement primaire, et se poursuivre jusqu'à la récidive ou le décès (voir Howell et al., 2011; Sussman et al., 2016), alors que d'autres, plus restreintes, n'incluent que la période de transition entre le traitement actif et les soins de première ligne (voir par exemple Chomik, 2010; Ward, Doll, Ristovski-Slijepcevic, Kazanjian et Golant, 2010). $\mathrm{Si}$ « tout le monde est un survivant », il devient difficile d'identifier les personnes dont la situation ou les besoins sont complexes, ce qui peut avantager les patients mieux outillés pour trouver réponse à leurs besoins, peu importe la complexité de leur cas ou les ressources d'autosoin. En outre, ces définitions viennent brouiller la distinction entre les personnes « guéries » qui mènent une vie relativement normale et celles qui vivent pendant des décennies avec un cancer stable mais métastatique (c.-à-d. non traitable), ou encore les survivants dont les soins nécessitent une approche palliative. Ainsi, les définitions les plus larges et politiquement correctes, semblent renforcer, d'une manière implicite et qui semble toute naturelle, le statut privilégié des patients dont le cancer est guérissable. Ces derniers contribuent ainsi au métarécit social, qui véhicule l'idée que l'expérience du cancer et la victoire sur la maladie sont un vecteur de transformation (Bell, 2013).

De plus, force est d'admettre que les documents disponibles traitent davantage des aspects collectifs et systémiques que des expériences individuelles. Nous avons en outre constaté que les termes «soins axés sur le patient » et « soins axés sur la personne » étaient facilement interchangeables, et nous avons été frappées par l'absence quasi totale de références à l'équité. Lorsque la question de l'équité est textuellement abordée, elle se limite souvent à l'amélioration de l'accès aux soins à l'échelle de la population (par exemple, pour les collectivités rurales), ou à la cueillette de données sur les déterminants sociaux (comme le statut socioéconomique ou le niveau d'éducation) pour décrire les inégalités collectives plus qu'individuelles. Par conséquent, les documents nous ont beaucoup appris sur la « vision » du système par rapport au principe d'équité, de même que sur les forces sociales à l'œuvre pour faire évoluer cette perception.

\section{Analyse secondaire de transcriptions d'entrevues}

Nous avons ensuite procédé à une analyse secondaire des données de 61 entrevues réalisées auprès de 19 adultes se décrivant comme des survivants au cancer. Il s'agissait d'une étude longitudinale de 500 entrevues menées auprès de 125 patients atteints de cancer sur une période de 7 ans (de 2005 à 2012); les entretiens mettaient l'accent sur la perception qu'avaient les patients de la communication entourant les soins du cancer (voir Thorne et Stajduhar, 2012; Thorne et al., 2014). Grâce à ces entrevues, nous avons pu mesurer la profondeur de l'incertitude qui habite les patients après la fin du traitement, lorsque le filet de sécurité du système de soins disparaît (souvent soudainement). Les patients se sentent « abandonnés » aux mains du système de soins de première ligne, leur cas relégué à des praticiens dont on les a inconsciemment encouragés à se méfier en leur disant qu'ils connaissaient mal le cancer, contrairement aux spécialistes. Non seulement le « retour à la normale » tant attendu leur échappe complètement, mais ils perdent souvent le précieux réseau de soutien social qui s'était constitué autour d'eux pendant la phase de traitements actifs. Lorsque les patients ne sont plus officiellement malades, il n'est pas rare que leur réseau de soutien s'effrite; dans certains cas, les amis et la famille s'attendent même à une reprise immédiate de toutes les responsabilités déléguées à d'autres depuis le diagnostic de cancer.

Les survivants rapportent aussi que le système et les professionnels de la santé insistent beaucoup sur les aspects biologiques et physiques de ce qui constitue, pour eux, une expérience holistique. De l'avis de nombreux patients, c'est l'absence ou la présence, voire la gravité de la maladie, de maladie, qui détermine la priorité d'accès aux soins pour les survivants. Par conséquent, lorsque les patients entrent en rémission ou qu'ils ne sont plus (ou presque plus) malades, ils se sentent exclus de ce système axé sur la pathologie. Selon eux, le système se désintéresse aussi des effets différés ou persistants du traitement, ainsi que des effets psychologiques post-traumatiques des expériences qui les ont transformés. Ce contexte leur a fait prendre conscience du fonctionnement général du système de santé, notamment de ses politiques hiérarchiques et des tensions qui existent entre les différentes sphères de compétence, mais aussi de ses moteurs d'efficacité. Cette constatation en a amené plusieurs à se sentir invisibles, eux qui se sentent encore très vulnérables par rapport au système de soins.

\section{Entrevues avec les survivants et les intervenants}

Une fois les résultats des deux premières étapes de l'étude obtenus, nous avons terminé cette étude en réalisant des entrevues en personne avec 34 survivants du cancer. Ces derniers ont été sélectionnés par échantillonnage intentionnel afin d'assurer une grande diversité de variables liées tant à la santé et qu'aux déterminants sociaux; 12 autres personnes ont aussi été recrutées pour leur expertise et leur expérience du développement et de la mise en œuvre des soins aux survivants du cancer au Canada. La moitié d'entre eux étaient des médecins, le reste du groupe étant constitué d'infirmières autorisées, d'infirmières praticiennes ou de professionnels de la santé psychosociale; chacun jouait un rôle dans le système de soins du cancer, que ce soit dans l'administration, l'éducation, la recherche ou la création de programmes sur la survivance. Sept de ces douze spécialistes provenaient de la ColombieBritannique et les autres, de l'Alberta ou de différents centres de traitement du cancer de l'Ontario. Les entrevues ont été menées par l'auteure principale [TT] sur une période de 18 mois (2016-2017) et ont permis d'amasser une foule de 
données sous forme de transcriptions, de notes d'observation et de notes d'analyse, toutes codées et classées à l'aide du logiciel d'analyse qualitative NVivoMC. En majorité, les entrevues des survivants ont révélé que leurs attentes avaient été déçues, les soins ne correspondant pas à la réalité qu'ils avaient imaginée et espérée. Nous avons donc orienté nos réflexions analytiques sur la nature et la variation des écarts séparant la perception des besoins et l'expérience réellement vécue, ainsi que sur la manière dont les participants à l'étude s'expliquaient ces écarts.

La plupart des survivants s'attendaient à recevoir des soins holistiques individualisés et axés sur la personne, dans le cadre d'une relation continue et réciproque avec les professionnels de la santé. La plupart s'attendaient à devoir prendre en charge une partie des soins, mais ils pensaient pouvoir compter sur le soutien et l'expertise des professionnels à cet égard. Nous avons constaté que certains déterminants sociaux de la santé bien précis jouaient un rôle de premier plan dans ces attentes. Par exemple, les gens des régions rurales ou isolées, les personnes souffrant d'un cancer « non traitable », rare ou complexe ou présentant des maladies chroniques concurrentes, les jeunes ou les adultes très âgés, de même que les patients parlant une langue autre que l'anglais ou appartenant à d'autres « groupes visés par l'équité » reconnaissables, s'attendaient beaucoup moins à ce qu'on réponde à leurs besoins particuliers comparativement à ce qu'ils pensaient qu'on ferait pour ce qu'ils percevaient comme un patient «typique ». En raison de la grande visibilité des ressources et du soutien destinés aux patientes atteintes de cancer du sein, les personnes souffrant d'un autre cancer avaient aussi l'impression d'être désavantagées. Quant aux personnes ayant déjà vécu une expérience négative dans le système de soins du cancer, elles étaient moins susceptibles de croire qu'elles auraient accès à des soins aux survivants répondant à leurs besoins. Ainsi, nous avons constaté à quel point les conditions initiales peuvent perpétuer les écarts en ce qui a trait aux attentes et à l'engagement des patients, ce qui peut accentuer les inégalités pour les personnes déjà vulnérables.

De nombreux participants à l'étude ont décrit tous les efforts déployés pour rebâtir leur vie après les traitements et pour obtenir des soins aux survivants; leurs récits nous ont surpris et nous ont aussi appris que les survivants se sentaient souvent tenus à l'écart des communications concernant leurs soins, l'information circulant d'un professionnel à l'autre sans qu'ils soient au courant. Par exemple, certains patients savaient qu'on leur avait fait un plan de survivance, mais ne l'avaient jamais vu. Dans l'ensemble, on comprend que si certains survivants trouvent leur compte dans les ressources et le soutien existants, ce n'est pas le cas de nombreux patients. Selon nos recherches, le sous-groupe de survivants du cancer le plus susceptible de voir ses attentes comblées de manière optimale était surtout constitué de femmes âgées de 50 à 70 ans au statut socioéconomique élevé souffrant d'un cancer du sein traitable et vivant en milieu urbain, qui disposaient notamment de bonnes prestations d'assurance-maladie et de soutien pour le retour au travail, d'un réseau social solide, d'une excellente capacité à pratiquer l'autosoin et à faire entendre leur voix, qui ressentaient peu d'effets secondaires persistants et qui entretenaient de bonnes relations avec leur médecin traitant. À certains égards, il semble le système de soins aux survivants soit majoritairement conçu pour répondre aux besoins de ce patient type.

De leur côté, les intervenants du système de soins aux survivants du cancer ont été en mesure de fournir une perspective éclairée sur le pourquoi et le comment de ces écarts, leur connaissance intime du système leur permettant une réflexion critique sur les structures, contextes et facteurs susceptibles de nuire à la qualité des soins aux survivants, mais aussi de favoriser l'équité et la qualité des services. Essentiellement, ils ont dit s'inquiéter des « critères de mesure » utilisés pour évaluer les inégalités potentielles, critères qui consistent à comparer les groupes à une « norme » standardisée plutôt que d'examiner les différentes circonstances individuelles d'un point de vue multidisciplinaire. Ils ont aussi attiré notre attention sur le fait que les données du « groupe visé par l'équité » masquaient la grande iniquité vécue par certaines personnes. De plus, ils ont décrit des travers qui mènent à des lignes directrices sur la survivance qui ne tiennent pas compte de la diversité des patients et de leurs besoins : manque de flexibilité du système, influence du colonialisme, domination du modèle biomédical occidental, mentalité de pénurie, priorité donnée aux pratiques fondées sur les données probantes, invisibilité des déterminants sociaux de la santé, « normes » établies en fonction des groupes de tumeurs. Aux obstacles engendrés par ces travers s'ajoute des complexités structurelles, dont le fait que les ressources allouées pour les soins du cancer sont directement liées au traitement en raison de la façon dont fonctionne la rémunération des médecins les prestations (prolongées) d'assurance-maladie. Un autre problème se situe dans la concentration des connaissances et des services spécialisés dans les établissements de soins, qui se traduit par une absence (ou une pénurie) de services dans la communauté. Enfin, les intervenants mentionnent aussi les contradictions qui opposent les décisions systémiques, ancrées dans les priorités biomédicales, et la philosophie des soins axés sur la personne. De leurs propos se dégage une forme de paternalisme qu'ils qualifient « d'arrogance institutionnelle » par rapport au nombre croissant de survivants du cancer et à la responsabilité de les soutenir.

\section{DISCUSSION}

Plusieurs de nos résultats confirment la pertinence de critiquer l'équité, un sujet de plus en plus abordé dans la littérature sur les soins du cancer ces dernières années (Truant, 2017). Nous croyons que la méthodologie multidimensionnelle à plusieurs niveaux adoptée pour la présente étude nous a permis de mettre en lumière les difficultés inhérentes à l'adoption, au Canada, d'une approche de soins aux survivants du cancer de grande qualité axée sur la population. Plus particulièrement, cette méthodologie nous amène à reconnaître non seulement le niveau relativement élevé de besoins non satisfaits de ce que nous appelons des « groupes visés par l'équité », mais également les nombreux et divers besoins des individus qui, pour toutes sortes de raisons, ne cadrent pas dans les normes 
dominantes. L'une des retombées les plus importantes de cette étude est sans contredit de jeter les bases d'une réflexion critique nécessaire sur la définition de la survivance dans le contexte de la médecine personnalisée, en pleine évolution, ainsi que sur les patients qui sont avantagés ou, au contraire, systématiquement désavantagés par les idées que nous entretenons ainsi que par les structures de soins aux survivants et les moyens actuellement mis en place pour déterminer qui doit recevoir des soins et quels types de soins sont appropriés.

Des voix s'élèvent toutefois pour faire entendre celles des survivants du cancer, ce qui nous semble très prometteur. À ce chapitre, soulignons l'importance toute particulière du récent appel national à se fonder sur l'expérience des patients qui vivent avec le cancer et qui survivent à la maladie pour guider les pratiques cliniques, les processus administratifs du système de santé, et les bases de données gouvernementales des provinces et des territoires (PCCC, 2018).

À la lumière de ces résultats, nous sommes conscients de la nécessité de porter une attention particulière aux discordances entre l'offre de services à l'échelle de la population et les soins nécessaires pour répondre aux besoins uniques et distinctifs des individus afin de relever les défis liés à la survivance au Canada, en écoutant notamment les patients et les professionnels, qui sont de plus en plus nombreux à revendiquer du changement. Pour ce faire, nous devrons repenser les modèles organisationnels ayant traditionnellement structuré et hiérarchisé les soins du cancer, et les soins de santé en général. Enfin, il faudra revoir le déploiement des ressources de soins, notamment le rôle des différentes professions dans la planification, la prestation et la direction des soins, particulièrement complexes, dédiés aux survivants.

\section{Recommandations pour l'amélioration du système}

Bien que les obstacles à l'équité et à la qualité des soins aux survivants du cancer soient nombreux et complexes, nous pouvons aussi en apprendre beaucoup en misant sur les forces du système qui sont rapportées dans la documentation, ainsi que dans les propos des survivants et des intervenants. Il nous faut aussi reconnaître que l'approche traditionnelle, qui met l'accent sur l'aspect curatif des soins du cancer, a abouti à un système axé sur la pathologie aiguë et épisodique plutôt que sur l'intégration des soins à la vie quotidienne des patients et des survivants, qui sont fort nombreux. Nous comprenons tous l'importance des traitements, mais si nous sommes sincères dans notre idéal d'offrir des soins axés sur la personne, la gestion de la maladie ne doit pas se faire aux dépens des besoins psychologiques, émotionnels, spirituels et informationnels du patient (PCCC, 2018). Et bien que les lignes directrices fondées sur les données probantes soient extrêmement utiles au moment de concevoir et de dispenser les traitements, elles occultent nécessairement la pertinence des différences individuelles (Thorne et Sawatzky, 2014). Par conséquent, nous organisons nos systèmes de soins en fonction de la « personne moyenne » en mettant de côté la diversité et le contexte, car nos approches reposent uniquement sur des critères normatifs (Sulik, 2013). Nous devons éviter d'adhérer aveuglément à des idéologies organisationnelles, qu'il s'agisse de pratiques fondées sur les données probantes ou de soins axés sur la personne, et tenir compte du fait que ces idéologies peuvent entrer en conflit les unes avec les autres, sachant que les patients en feront les frais si nous ne collaborons pas pour résoudre en priorité les enjeux complexes et très concrets de l'iniquité.

Nous savons que le fait de définir les standards des soins aux survivants du cancer en privilégiant les normes eurocentriques est une pratique colonialiste ayant des répercussions réelles sur les personnes qui n’entrent pas dans le moule théorique (Anderson et al., 2009). Nous aurions tout avantage à impliquer davantage les groupes visés par l'équité et à profiter de leurs commentaires constructifs pour déterminer quelles sont nos préconceptions des soins qui desservent systématiquement différents groupes de survivants. Nous devons aussi apprendre à écouter l'avis réfléchi des gens qui sont au cœur de l'action, notamment les décideurs du système de santé et les cliniciens, sur certains sujets tels que les groupes moins bien servis par nos établissements et services de soutien. Nous disposons déjà d'outils précieux pour bien nous préparer à accueillir toute la diversité individuelle avec laquelle nos systèmes doivent inévitablement composer si nous voulons favoriser l'émergence de systèmes qui pourront fournir des soins d'excellente qualité aux groupes minoritaires les plus vulnérables.

Nous savons, grâce aux survivants du cancer, qu'une communication efficace et une bonne relation avec les professionnels de la santé sont la clé pour aplanir les relations hiérarchiques que les patients perçoivent comme un obstacle aux soins. En voyant nos systèmes comme des incubateurs de relations et non comme des établissements dépersonnalisés qui ne servent que d'unités de services, nous pourrons concentrer nos efforts à combler les lacunes et à satisfaire les besoins des patients. La continuité des soins fait depuis longtemps partie des idéaux de la pratique infirmière et nous devons continuer de défendre notre capacité à nouer des liens continus et à assurer un transfert des soins efficace du point de vue relationnel. Lorsque les critères de mesure et la quantification des facteurs complexes de la qualité de vie éclipsent l'aspect relationnel des soins, il va de soi que cet aspect cesse d'être considéré comme une priorité. Nous devons donc trouver des manières de remettre la communication au centre de la trajectoire des soins du cancer, non seulement pour assurer la sécurité des patients, mais aussi pour nous assurer de placer la participation des patients au cœur de nos approches de soins.

Pour faire évoluer les soins aux survivants, nous devons envisager l'adoption de modèles de soins stratifiés en fonction de la complexité des besoins. Cela présuppose que nous connaissions les besoins de nos patients et que nous créions ensemble des approches de soins, avec la collaboration active des intervenants et des survivants. Nous croyons que de confier aux infirmières la direction des soins aux survivants offre de grandes possibilités. Pour ce faire, il serait notamment possible d'embaucher des infirmières spécialisées en oncologie (inf. aut. ou IP) dans les milieux de soins de première ligne, en modifiant les modèles en place en fonction 
des préoccupations actuellement criantes dans le domaine de la survivance, mais également de celles à venir. Évidemment, pour apporter de tels changements, il faudrait modifier la structure en place et la culture institutionnelle, notamment en ce qui a trait au rôle joué par les infirmières dans de nombreuses organisations de soins du cancer. Plutôt que d'organiser les soins en fonction des groupes de tumeurs, comme le préconise le modèle biomédical traditionnel, d'ailleurs mis à mal par la médecine de précision et les nouvelles philosophies de la prise en charge du cancer, nous devrions militer en faveur d'une organisation fondée sur les expériences et les besoins des patients intégrant des services comme le soutien en cas de maladies chroniques ainsi que l'approche palliative des soins, qui sous-tendent des objectifs de soins individualisés. Les modèles de soins menés par les pairs auront leur rôle à jouer, mais puisqu'ils fonctionnent mieux pour certains groupes de survivants que d'autres, ils ne peuvent remplacer une véritable intégration des soins. De plus, nos modèles doivent proposer des ressources facilement accessibles pour le retour au travail, le soutien familial et le soutien communautaire.

Pour tirer parti des possibilités qui s'offrent à nous, nous allons évidemment devoir miser sur la sensibilité culturelle (par un accueil respectueux et déférent de la culture de l'autre), un concept qui devra faire l'objet de formations et auquel tous les professionnels de la santé devront être sensibilisés, qu'ils soient du domaine des soins spécialisés ou des soins de première ligne. Nous allons aussi devoir soutenir les communautés de pratique grâce auxquelles les professionnels de la santé peuvent continuer d'enrichir leur compréhension de la complexité des soins aux survivants afin de maintenir leur engagement à cet égard.

\section{RÉFÉRENCES}

Ahmed, S., \& Shahid, R.K. (2012). Disparity in cancer care: A Canadian perspective. Current Oncology, 19(6), e376.

Anderson, J., Rodney, P., Reimer Kirkham, S., Browne, A.J., Khan, K.B., \& Lynam, M.J. (2009). Inequities in healthcare viewed through the ethical lens of critical social justice: Contextual knowledge for the global priorities ahead. Advances in Nursing Science, 32(4), 282-294.

Ashing-Giwa, K., Betts-Turner, B., Carter, D., Rosales, M., Jackson, P.A., Tapp, C., ... Martin, V. (2013). Are survivorship care plans responsive to African-American breast cancer survivors? Voices of survivors and advocates. Journal of Cancer Survivorship, 7(3), 283291. doi:10.1007/s11764-013-0270-1

Association canadienne des infirmières en oncologie (CANO/ ACIO) (2006). Practice standards and competencies for the specialized oncology nurse. [en ligne] https://cdn.ymaws.com/ www.cano-acio.ca/resource/resmgr/standards/CONEP_ Standards2006September.pdf

Association des infirmières et infirmiers du Canada (2017). Code of ethics. Ottawa, Ontario. [en ligne] https://www.cna-aiic.ca/-/media/ cna/page-content/pdf-en/code-of-ethics-2017-edition-secureinteractive.pdf

\section{Vision d'avenir}

Pour arriver à dispenser des soins de grande qualité aux survivants du cancer en toute équité, nous devrons poursuivre les efforts de recherche et de sensibilisation. Voici deux des grandes questions qui devront orienter notre recherche de modèles de soins évolutifs : a) Comment et où intégrer les difficultés psychologiques, sexuelles, sociales et financières que vivent les survivants et qui caractérisent plusieurs de leurs besoins de première nécessité? et b) Comment articuler des lignes directrices pour l'ensemble de la population qui n'évinceront pas les préoccupations immédiates et très concrètes des individus, et particulièrement des personnes les plus vulnérables? Le Partenariat canadien contre le cancer a clairement décrit la voie que doit suivre le Canada :

Entre le moment où l'on commence à se demander si on est atteint d'un cancer et les soins de suivi après le traitement, il s'agit d'une expérience continue pour toutes les personnes concernées. L'offre de soins centrés sur la personne à chaque étape de cette expérience permet de considérer les patients et les membres de leur famille comme des personnes uniques, de leur prodiguer des soins coordonnés qui prennent en compte leur temps et leurs préférences, de leur donner accès à des renseignements utiles qui favorisent l'autonomie en matière de soins, et de combler leurs différents besoins à mesure qu'ils se présentent (PCCC, 2018, p. 45).

Pour arriver à offrir à l'ensemble de la population canadienne des soins aux survivants de grande qualité, nous devons aligner les recherches en cours avec la riche sagesse expérientielle et clinique des professionnels responsables de la prestation des services au quotidien. Nous devons aussi travailler de concert pour porter à l'attention des planificateurs et des décideurs les réflexions critiques qui leur permettront de suivre les progrès et diagnostiquer les lacunes.

Bell, K. (2010). Cancer survivorship, mor(t)ality and lifestyle discourses on cancer prevention. Sociology of Health Q Illness, 32(3), 349-364. doi:10.1111/j.1467-9566.2009.01198.x

Bell, K. (2012). Remaking the self: Trauma, teachable moments, and the biopolitics of cancer survivorship. Culture, Medicine, and Psychiatry, 36(4). 584-600.

Bell, K. (2013). Biomarkers, the molecular gaze and the transformation of cancer survivorship. Biosocieties, 8(2), 124-143. doi:10.1057/ biosoc. 2013.6

Bell, K., \& Ristovski-Slijepcevic, S. (2013). Cancer survivorship: Why labels matter. Journal of Clinical Oncology: Official Journal of the American Society of Clinical Oncology, 31(4), 409.

Boehmer, U., Glickman, M., Milton, J., \& Winter, M. (2012). Healthrelated quality of life in breast cancer survivors of different sexual orientations. Quality of Life Research, 21(2), 225-236.

British Columbia Ministry of Health (2015). The British Columbia patientcentred care framework. [en ligne] http://www.health.gov.bc.ca/library/ publications/year/2015_a/pt-centred-care-framework.pdf

Brooks, C. (2010). "I just didn't tell anybody what I was doing": Aboriginal women cancer survivors visualize social support. Canadian Woman Studies, 28(2/3), 63-70. 
Chomik, T.A. (2010). Supporting the role of primary care in cancer follow-up. (A Discussion Paper). Vancouver, Canada: Canadian Association of Provincial Cancer Agencies.

Epstein, R.M., Fiscella, K., Lesser, C.S., \& Stange, K.C. (2010). Why the nation needs a policy push on patient-centered health care. Health Affairs (Project Hope), 29(8), 1489-1495.

Falk-Rafael, A. (2005). Speaking truth to power: Nursing's legacy and moral imperative. Advances in Nursing Science, 28(2), 212-223.

Feuerstein, M., \& Ganz, P. (2011). Quality health care for cancer survivors. In M. Feuerstein \& P. Ganz, Eds., Health services for cancer survivors: Practice, policy and research (pp. 373-383). New York, NY: Springer. doi:10.1007/978-1-4419-1348-7_18

Fitch, M., Zomer, S. Lockwood, G., Louzado, C., Moxam, R.S., Rahal, R., \& Green, E. (2018 in press). Experiences of adult cancer survivors in transitions. Supportive Care in Cancer. Retrieved from https:// doi.org/10.1007/s00520-018-4605-3

Gifford, W., Thomas, R., Barton, G., Grandpierre, V., \& Graham, I.D. (2018). "Breaking the silence" to improve cancer survivorship care for First Nations peoples: A study protocol for an indigenous knowledge translation strategy. International Journal of Qualitative Methods, 17, 1-6, https://doi.org/10.1177/1609406918774133

Hankivsky, O. (2011). Health inequities in Canada: Intersectional frameworks and practices. Vancouver, BC: UBC Press.

Howell, D., Green, E., Jones, G.W., Jones, J. M., Parkinson, M., Payeur, N., ... Cancer Journey Survivorship Expert Panel. (2011). Survivorship services for adult cancer populations: A panCanadian guideline. Current Oncology (Toronto, Ont.), 18(6), e265e281. doi:10.3747/co.v18i6.956

Jones, J., \& Grunfeld, E. (2011). Specific challenges in optimizing health care for cancer survivors. In M. Feuerstein \& P.A. Ganz (Eds.), Health Services for Cancer Survivors (pp. 3-25). Bethesda, MD: Springer. doi:10.1007/978-1-4419-1348-7_1

Kromm, E.E., Smith, K.C., \& Singer, R.F. (2007). Survivors on cancer: The portrayal of survivors in print news. Journal of Cancer Survivorship, 1(4), 298-305. doi:10.1007/s11764-007-0033-y

Lotfi-Jam, K., Schofield, P., \& Jefford, M. (2009). What constitutes ideal survivorship care? Cancer Forum, 33(3), 172-175.

Malin, J. (2011). What is quality health care for cancer survivors? In M. Feuerstein \& P. Ganz (Eds), Health services for cancer survivors: Practice, policy and research (pp. 27-49). New York, NY: Springer. doi:10.1007/978-1-4419-1348-7_2

Mehnert, A., de Boer, A., \& Feuerstein, M. (2013). Employment challenges for cancer survivors. Cancer, 119 Suppl 11(11), 2151-2159. doi:10.1002/cncr.28067

National Academies of Sciences, Engineering, and Medicine. (2018). Long-term survivorship care after cancer treatment: Proceedings of a workshop. Washington DC: National Academies Press. doi:https:// doi.org/10.17226/25043.

Ness, S., Kokal, J., Fee-Schroeder, K., Novotny, P., Satele, D., \& Barton, D. (2013). Concerns across the survivorship trajectory: Results from a survey of cancer survivors. Oncology Nursing Forum, 40(1), $35-42$.

Niu, X., Roche, L. M., Pawlish, K.S., \& Henry, K.A. (2013). Cancer survival disparities by health insurance status. Cancer Medicine, 2(3), 403-411. doi:10.1002/cam4.84

Partenariat canadien contre le cancer (PCCC) (2012). Advances in survivorship care: Resources, lessons learned and promising practices. Toronto (Ontario).

Partenariat canadien contre le cancer (PCCC) (2018). Living with cancer: A report on patient experience. Toronto (Ontario).

Réseau universitaire de santé / University Health Network (UHN), Princess Margaret Hospital (PMH) (s.d.). Electronic living laboratory in cancer survivorship research (ELLICSR). [en ligne] https://www.ellicsr.ca/en/Pages/default.aspx
Resnick, M.J., Lacchetti, C., Bergman, J., Hauke, R.J., Hoffman, K.E., Kungel, T.M., \& Penson, D.F. (2015). Prostate cancer survivorship care guideline: American Society of Clinical Oncology clinical practice guideline endorsement. Journal of Clinical Oncology, 33(9), 1078-1085. doi:10.1200/JCO.2014.60.2557

Ristovski-Slijepcevic, S. (2008). Environmental scan of cancer survivorship in Canada: Conceptualization, practice and research. Vancouver BC: Canadian Partnership Against Cancer. [en ligne] http://www.partnershipagainstcancer.ca/wpcontent/uploads/ Survivorship_Svetlana_Ristovski-Slijepcevic.pdf

Shaw, A.E. \& Jennings-Shaw, P.J. (2017). Surveillance or symptom management? Insights from 5 years of caring for survivors. Journal of Clinical Oncology, 35(5 Suppl.), 44-44. doi:10.1200/ JCO.2017.35.5_suppl.44

Sinding, C. (2014). Who gets better cancer care? Differences between people result in differences between treatment - and outcomes. The Spectator, pp. A.15.

Skinner, D. (2012). The gendering of cancer survivorship. Health, Culture and Society, 3(1), 63-76.

Smith, J.L., \& Hall, I.J. (2015). Advancing health equity in cancer survivorship: Opportunities for public health. American Journal of Preventive Medicine, 49(6), S477-S482.

Sulik, G. (2013). What cancer survivorship means. Virtual Mentor, 15(8), 697-703.

Sussman J., Varela, N., Cheung, M., Hicks, L., Kraftcheck, D., Mandel, J., ... McQuillan, R. (2016). Follow-up care for survivors of lymphoma who have received curative-intent treatment. Current Oncology, 23(5), e499-e513.

Thorne, S. (2016). Interpretive description: Qualitative research for applied practice (2nd ed.). New York \& London: Routledge.

Thorne, S., Hislop, T.G., Kim-Sing, C., Oglov, V., Oliffe, J.L., \& Stajduhar, K.I. (2014). Changing communication needs and preferences across the cancer care trajectory: Insights from the patient perspective. Supportive Care in Cancer, 22(4), 1009-1015.

Thorne, S., \& Sawatzky, R. (2014). Particularizing the general: Sustaining theoretical integrity in the context of an evidencebased practice agenda. Advances in Nursing Science, 37(1), 5-18. doi:10.1097/ANS.0000000000000011

Thorne, S.E., \& Stajduhar, K.I. (2012). Patient perceptions of communications on the threshold of cancer survivorship: Implications for provider responses. Journal of Cancer Survivorship, 6(2), 229-237. doi:10.1007/s11764-012-0216-z

Thorne, S., Stephens, J., \& Truant, T. (2016). Building qualitative study design using nursing's disciplinary epistemology. Journal of Advanced Nursing, 72(2), 451-460.

Truant, T. (2017). Equity in cancer care: Strategies for oncology nurses. Nursing Clinics of North America, 52, 211-225.

University Health Network (UHN), Princess Margaret Hospital $(\mathrm{PMH})$ (n.d.). Electronic living laboratory in cancer survivorship research (ELLICSR). Retrieved from https://www.ellicsr.ca/en/ Pages/default.aspx

Ward, A., Doll, R., Ristovski-Slijepcevic, S., Kazanjian, A. \& Golant, M. (2010). Cancer transitions: A supportive care program for cancer survivors. Oncology Exchange, 9, 12-14.

Weaver, K.E., Geiger, A.M., Lu, L., \& Case, L.D. (2013). Rural-urban disparities in health status among US cancer survivors. Cancer, 119(5), 1050-1057.

Wittal, D.M. (2018). Bridging the gap from the oncology setting to community care through a cross-Canada environmental scan. Canadian Oncology Nursing Journal, 28(1), 38-45. 